\title{
Per- and polyfluoroalkyl substances (PFAS)—contaminants of emerging concern
}

\author{
Erin S. Baker ${ }^{1}$. Detlef R. U. Knappe ${ }^{2}$
}

Accepted: 26 November 2021 / Published online: 2 December 2021

c) Springer-Verlag GmbH Germany, part of Springer Nature 2021

Per- and polyfluoroalkyl substances (PFAS) are a family of persistent organic pollutants of global concern due to correlations between human exposure and adverse health outcomes including organ damage, cancers, decreased fertility, and impacts on the immune system. PFAS are highly fluorinated anthropogenic chemicals first developed in the 1940s during the Manhattan Project to enrich uranium. Due to their unique and highly stable carbon-fluorine bonds, PFAS possess distinct physicochemical properties such as resistance to grease, oil, water, and heat. These characteristics therefore make PFAS intrinsically suited for many household and industrial applications. To date, PFAS have been incorporated into commercial products including nonstick cookware, food packaging, stain- and water-resistant fabrics, carpeting, cleaning products, paints, and firefighting foams, just to name a few. However, over the last 50 years and mainly the last decade, increasing concerns pertaining to PFAS persistence in both the environment and human body have arisen. For example, some legacy PFAS have even been detected in samples from the 1950s, showing they are still present 70 years later. Additionally, current estimates suggest many PFAS have half-lives of years in the human body, which is greatly concerning for both the bioaccumulation and biomagnification of the whole family of molecules. PFAS are also readily found in drinking water and many food products, and their detection in the blood of $>98 \%$ of

Published in the topical collection Per-and Polyfluoroalkyl Substances (PFAS) - Contaminants of Emerging Concern with guest editors Erin Baker and Detlef Knappe.

Erin S. Baker

ebaker@ncsu.edu

$\triangle$ Detlef R. U. Knappe

knappe@ncsu.edu

1 Department of Chemistry, North Carolina State University, Raleigh, NC 27695, USA

2 Department of Civil, Construction, and Environmental Engineering, NC 27695 Raleigh, USA humans studied for their presence is alarming. While the production of legacy PFAS such as perfluorooctanoic acid (PFOA) and perfluorooctane sulfonic acid (PFOS) has been discontinued in certain parts of the world, their replacement species, termed emerging PFAS, are currently being synthesized to be less bioaccumulative yet still provide the desired characteristics necessary in many applications. While it was hoped that adding an ether linkage or other chemical modifications would make the replacements more degradable, recent publications have shown that this is not the case. GenX, for example, is as equally persistent as PFOA and PFOS under very harsh oxidizing conditions. Additionally, the toxicity of these emerging species is still vastly unknown, so improved analytical evaluations and monitoring capabilities are essential to determine their effects on the environment and human health. Furthermore, PFOA, PFOS, and many other legacy PFAS are routinely detected in human blood and the environment due to their chemical persistence, so these legacy PFAS must also be evaluated alongside the emerging species. The simultaneous detection of both emerging and legacy PFAS however causes challenges and is driving many current analytical developments.

To date, the Environmental Protection Agency (EPA) has estimated over 9000 different PFAS exist including the parent PFAS and their metabolic and degradation products. Therefore, over the last decade, concerns about the numerous PFAS species and adverse human health connections have continued to mount. Recently movies such as The Devil We Know and Dark Waters have brought the history of PFAS and their toxic attributes to the forefront of public awareness. Furthermore, in October 2021, both John Oliver and Trevor Noah had special segments highlighting PFAS presence and related health effects. We feel these broadcastings are essential to continue educating both the scientific community and the general public of the PFAS epidemic and corrective measures needed at both the state and federal level. We therefore determined that it was imperative to assemble this PFAS topical collection to be a critical resource as 
the field continues its forward momentum. Specifically, the articles included in this topical collection detail evolving and innovative analysis strategies for PFAS assessments and range from PFAS detection in processed foods, dairy milk, and human plasma to novel instrumental platforms and data analysis tools for the assessment of unknown PFAS. We believe this topical collection highlights the current state of the field and showcases certain directions that PFAS research must continue towards to advance our knowledge of PFAS toxicity, persistence, and remediation for both the legacy and emerging species.

Publisher's Note Springer Nature remains neutral with regard to jurisdictional claims in published maps and institutional affiliations.

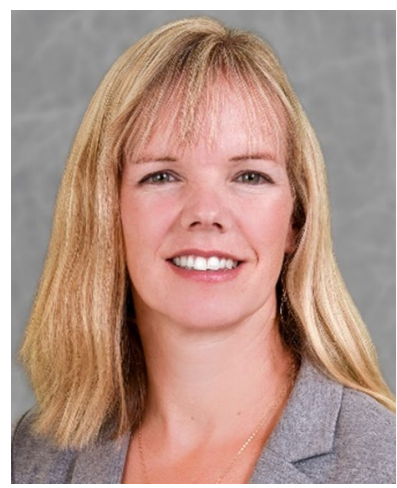

Erin S. Baker is Associate Professor at North Carolina State University in Raleigh, NC. To date, she has published over 140 peerreviewed papers utilizing ion mobility spectrometry in conjunction with mass spectrometry (IMS-MS) to study both environmental and biological systems. She has received seven US patents and two R\&D 100 Awards, aided in the commercialization of the Agilent 6560 IMS-QTOF MS, and was a recipient of the 2016 ACS Rising Star Award for Top Midcareer Women Chemists. She was also named on both the 2019 and 2021 Analytical Scientist's "Top 100 Power List."

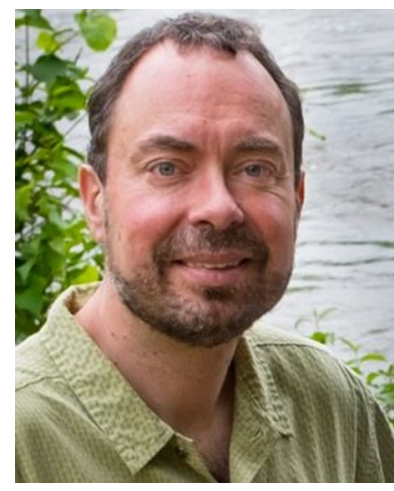

Detlef Knappe is the S. James Ellen Distinguished Professor of Civil, Construction, and Environmental Engineering at North Carolina State University. $\mathrm{He}$ is also Deputy Director of the NC State Superfund Center which is evaluating "Environmental and Health Effects of PFAS" and a member of the Center for Human Health and the Environment. He is interested in drinking water quality and treatment, water reuse, organic micropollutants, development of water treatment processes for polar and persistent organic pollutants, and the fate of organic pollutants in solid waste landfills. He is a Trustee of the American Water Works Association's (AWWA's) Water Science and Research Division, and he is a member of the North Carolina Secretaries' Science Advisory Board. He also serves on the AWWA's Organic Contaminants Research Committee and the Standards Committee for Activated Carbon. 